Received: 11.10 .2019

Revised: 15.11 .2019

Accepted: 20.12 .2019

DOI: $10.17804 / 2410-9908.2019 .6 .006-014$

\title{
EVALUATION OF STRESSES IN A PRESURIZED VESSEL BY MAGNETOELASTIC DEMAGNETIZATION
}

\author{
S. M. Kulak ${ }^{\text {a)* }}$, V. F. Novikov ${ }^{\text {b) }}$, and D. D. Mitrofanov ${ }^{\text {c) }}$ \\ Tyumen Industrial University, \\ 38, Volodarskogo St., Tyumen, 625000, Russian Federation \\ a) (iD 0000-0002-5970-8893 ksm-rabochi@ rambler.ru; \\ b) (iD 0000-0002-1987-351X \\ *Corresponding author. E-mail: ksm-rabochi@rambler.ru \\ Address for correspondence: ul. Melnikaite, 70, Tyumen, 625039, Russian Federation
}

Magnetoelastic demagnetization of the wall of a Propane gas cylinder undergoing tensile strains in two orthogonal directions under the influence of water pressure from the inside is studied. The magnetoelastic sensitivity of the anisotropic residual magnetization and the coercive force of a complexly loaded steel wall to elastic stresses is determined.

Keywords: stressed state of steel, magnetization, magnetoelastic demagnetization, magnetic stray field of residually magnetized steel.

\section{References}

1. Standart GOST R 56542-2015: Non-destructive testing. Classification of types and methods. Moscow, Standartinform Publ., 2019. (In Russian).

2. Gorkunov E.S., Yakushenko E.I., Zadvorkin S.M., Mushnikov A.N. The effect of elastic de-formations on the magnetic properties of chromium-Nickel steels. Physics of Metals and Metallography, 2015, vol. 116, no. 2, pp. 156-164. DOI 10.7868/S0015323015020072.

3. Nerazrushayushchiy control: spravochnik, Magnitnye metody kontrolya, t. 6, kn. 1 [Nondestructive Testing: A Handbook in 7 vols, V.V. Klyuev, ed., Klyuev V.V., Muzhitskiy V.F., Gorkunov E.S., and Shcherbinin V.E. Magnetic Testing Methods, vol. 6, book 1]. Moscow, Mashinostroenie Publ., 2004, 832 p. (In Russian).

4. Klyuev V.V., Muzhitskiy V.F., Gorkunov E.S., and Shcherbinin V.E. Nerazrushayushchiy control. Magnitnye metody kontrolya, kn.1, t. 6 [Nondestructive Testing, Magnetic Testing Methods, book 1, vol 6]. Moscow, Mashinostroenie Publ., 2006, 375 p. (In Russian).

5. Birss R.B., Fauncer C.A., Isaak E.D. Magneto-mechanical effects in iron and iron-carbon alloys. J. Appl. Phys., 1971, vol. 4, pp. 1040-1048. DOI: 10.1088/0022-3727/4/7/322.

6. Atherton D.L., Jiles D.C. Effects of stress on the magnetization of steel. IEEE Trans. Magn., 1983, vol. 19, no. 5, pp. 20212023. DOI: 10.1109/TMAG.1983.1062784.

7. Novikov V.F., Yatsenko T.A., Bakharev M.S. Rus. J. Nondestr. Test., 2001, vol. 37, no. 11, pp. 799-804; Rus. J. Nondestr. Test., 2002, vol. 38, no. 4, pp. 231-237.

8. Kostin V. N., Tsarkova T. P., Nichipuruk A. P., Loskutov V. E., Lopatin V. V., Kostin K. V. Irreversible Changes in the Magnetization as Indicators of Stress-strain State of Ferromagnetic Objects. Rus. J. Nondestr. Test., 2009, vol. 45, no. 11, pp. 786-798. DOI: 10.1134/S1061830909110059.

9. Zakharov V.A., Ul'yanov A.I., Gorkunov E.S. Regularities of the change in the coercive force under biaxial asymmetric deformation of steel 3. Russian Journal of Nondestructive Testing, 2010, vol. 46, no. 3, pp. 194-205. DOI: 10.1134/S1061830910030071. 
10. Novikov V.F., Sorokina S.V., Kudryashov M.E., Zakharov V.A., and Ul'yanov A.I., The influence of biaxial elastic deformation on the coercive force and local remanent magnetization of construction steels. Russ. J. Nondestr. Test., 2010, vol. 46, no. 7, pp. 520-526. DOI: $10.1134 / \mathrm{S} 1061830910070065$.

11. Kulak S.M., Novikov V.F., Probotyuk V.V., VatsenkovS. M., Fursov E.S. Magnetic Testing of Stressed State of Hydrotested Gas-Separator Wall. Russian Journal of Nondestructive Testing, 2019, vol. 55, no. 3, pp. 225-232. DOI: 10.1134/S1061830919030070.

12. Muzhitskii V.F., Popov B.E., and Bezlyud'ko G.Ya. Magnetic Measurements of StressedStrained States and Remaining Service Lives of Steel Structures in Hoisting Machines and Pressurized Vessels. Russ. J. Nondestr. Test., 2001, vol. 37, no. 1, pp. 29-36.

13. Zakharov V.A., Ul'yanov A.I., and Gorkunov E.S. Coercive Force of Ferromagnetic Steels under Biaxial Symmetric Tension of Materials. Rus. J. Nondestr. Test., 2011, vol. 47, no. 6, pp. 359-368. DOI: 10.1134/S1061830911060106.

14. Novikov V.F., Muratov K.R., Kulak S.M., Sorokina S.V., Ustinov V.P., Radchenko A.V. On controlling stresses in a complexly loaded steel construction by magnetoelastic demagnetization. Russian Journal of Nondestructive Testing, 2016, vol. 52, no. 6, pp. 357-361. DOI: $10.1134 / \mathrm{S} 1061830916060073$.

15. Novikov V.F., Prilutsky V.V. The properties of stripe-shaped residual magnetization and the possibilities for its application for nondestructive testing. Russian Journal of Nondestructive Testing, 2014, vol. 50, pp. 396-401. DOI: 10.1134/S1061830914070080.

16. GOST-15860. Steel welded cylinders for liquefied hydrocarbon gases at pressure up to 1.6 MPa. Specifications. Moscow, IPK Izdatelstvo standartov Publ., 1984. (In Russian).

17. Borodavkin P.P. and Sinyukov A.M. Prochnost' magistral'nykh truboprovodov [Strength of the Main Pipelines]. Moscow, Nedra Publ., 1984. (In Russian).

18. Vonsovskii S.V. and Shur Ya.S. Ferromagnetizm [Ferromagnetism]. Moscow, OGIZ Publ., 1948. (In Russian). 
Подана в журнал: 11.10 .2019

УДК 620.179.14

DOI: $10.17804 / 2410-9908.2019 .6 .006-014$

\title{
ОЦЕНКА НАПРЯЖЕНИЙ В СОСУДЕ С ДАВЛЕНИЕМ ПУТЕМ МАГНИТОУПРУГОГО РАЗМАГНИЧИВАНИЯ
}

\author{
С. М. Кулак ${ }^{\text {a)* }}$, В. Ф. Новиков ${ }^{\text {б) }}$, Д. Д. Митрофанов ${ }^{\text {в) }}$ \\ ФГБОУ Тюменский индустриальный университет, \\ 38, ул. Володарского, г. Тюмень, Российская Федерация \\ a) (iD 0000-0002-5970-8893 ksm-rabochi@ rambler.ru; \\ b) (iD 0000-0002-1987-351X \\ *Ответственный автор. Электронная почта: ksm-rabochi@ rambler.ru \\ Адрес для переписки: г. Тюмень, ул. Мельникайте, 70, Российская Федерация
}

Проведены исследования магнитоупругого размагничивания стенки газового баллона «Пропан», испытывающей деформации растяжения в двух ортогональных направлениях под действием давления воды изнутри. Определена магнитоупругая чувствительность анизотропной остаточной намагниченности и коэрцитивной силы сложнонагруженной стальной стенки к упругим напряжениям.

Ключевые слова: напряженное состояние стали, намагничивание, магнитоупругое размагничивание, магнитное поле рассеяния остаточно-намагниченной стали.

\section{1. Введение}

Изменение параметров магнитного гистерезиса сталей при их механической деформации является физической основой одноименных приборных методов неразрушающего контроля напряженно-деформированного состояния конструкций, изготавливаемых из них [1-6].

Напряженно-деформированное состояние (НДС) многих изделий и конструкций из ферромагнитных материалов (сталей) формируется под действием не только их одноосной деформации. Чаще всего многие стальные конструкции находятся в сложнонапряженном состоянии, когда они одновременно испытывают двух- или трехосные нагрузки, действующие вдоль взаимно-перпендикулярных направлений. Такая ситуация встречается, например, при эксплуатации трубопроводов, сосудов высокого давления, стенки которых испытывают сложнонапряженное состояние (двухосную упругую и пластическую деформацию). Однако при этом магнитоупругое поведение ферромагнитных материалов реальных конструкций под действием симметричных и ассиметричных напряжений мало изучено [7-11].

В плане изучения и разработки магнитных методов неразрушающего контроля напряженного состояния, актуальным является исследование магнитоупругих явлений не только на модельных образцах, подвергаемых в лабораторных условиях одноосным деформациям $[7,8]$, но и на реальных стальных конструкциях, испытывающих при эксплуатации многоосные деформации [9-14].

Цель работы - определение эквивалентных напряжений в стенке сосуда с давлением по магнитоупругому размагничиванию анизотропной локальной намагниченности. 
Задачи исследования:

- определение компонент напряжений в сложнонагруженной стальной конструкции;

- исследование зависимости анизотропии напряженности магнитного поля рассеяния локальной остаточной намагниченности стали и коэрцитивной силы от уровня напряжений, создаваемых двухосной деформацией.

В работе [11] изложены результаты исследования магнитоупругого размагничивания стенки газового сепаратора, испытывающей воздействие давления воды изнутри конструкции. Такое воздействие на стенку создавало в ней напряженное состояние, которое можно считать двухосным. При этом намагничивание стенки в виде двух пар осевых полос N-S осуществлялось только вдоль действия кольцевых напряжений. Это позволило обнаружить неоднородное по величине изменение напряженности магнитного поля рассеяния в разных точках остаточно-намагниченной стенки газового сепаратора при увеличении внутреннего давления до $18 \mathrm{MПа,} \mathrm{что} \mathrm{объясняется} \mathrm{особенностями} \mathrm{его} \mathrm{конструкции} \mathrm{и} \mathrm{термической} \mathrm{обра-}$ ботки. Поэтому актуальным явилось исследование и количественное сравнение магнитоупругого размагничивания сложнонагруженной конструкции, находящейся в однородном напряженном состоянии, при ее намагничивании вдоль каждого направления действующих деформаций.

\section{2. Материалы и методика исследований}

Исследования проводились на газовом баллоне «Пропан» объемом 50 л, состоящем из тонкостенной цилиндрической обечайки (высота баллона 1015 мм, внешний диаметр 299 мм, толщина стенки 3 мм) и выпуклых торцевых стенок в виде полусфер, изготовленных из стали Ст3 [16]. Баллон рассчитан на рабочее внутреннее давление газа 1,6 МПа, испытательное давление 2,5 МПа, разрушающее - не менее 5 МПа. Выбор такого объекта исследования обусловлен его простейшим устройством, необходимыми (достаточными) геометрическими размерами, а также возможностью создания в его стенке ассиметричного напряженного состояния относительно двух ортогональных направлений. При заполнении такого сосуда водой под давлением его боковая стенка будет испытывать в каждой точке неоднородные деформации растяжения вдоль кольцевой и осевой линий [17]. Компонентой напряжений, перпендикулярной поверхности боковой стенки баллона, можно пренебречь ввиду ее малости, поэтому для решения поставленных в исследовании задач по магнитоупругому размагничиванию сложнонагруженной конструкции можно ограничиться двухосными, а не трехосными напряжениями. Для определения величины ортогональных деформаций стенки газового баллона в работе использовался тензометрический метод.

В ходе проводимых исследований баллон заполнялся водой и соединялся с ручным опрессовщиком RP 30, с помощью которого создавалось внутреннее давление не более 3,5 МПа. На поверхность боковой стенки баллона в трех равноудаленных друг от друга точках одной осевой линии длиной 0,5 м наклеивались по два константановых тензорезистора для измерения ее кольцевой $\varepsilon_{\mathrm{K}}$ и осевой $\varepsilon_{0}$ деформации растяжением. Соответствующие механические напряжения растяжения $\sigma_{\text {к }}$ и $\sigma_{\text {o }}$, создаваемые ими в стенке баллона рассчитывались как для тонкостенной оболочки, а эквивалентные $\sigma_{э к в}-$ по четвертой энергетической теории прочности [17]. В результате тензометрических исследований деформации боковой стенки баллона, вызванной давлением воды до $P=3$ МПа было установлено, что величина $\sigma_{\text {к }}$ в трех точках на ее поверхности одинакова и превышают в четыре раза $\sigma_{\text {о }}$ (рис. 1$)$. Поэтому можно считать напряженное состояние боковой стенки газового баллона однородным по величине. Четырехкратное превышение результатов измерения $\varepsilon_{\mathrm{K}}$ над $\varepsilon_{0}$ может быть вызвано наличием преобладающих остаточных внутренних механических напряжений в стали, ориентированных вдоль кольцевого направления в стенке, создаваемых после закручивании в цилиндр стального листа в процессе изготовления баллона и недостаточной термической обработки готового изделия после сборки.

Kulak S. M., Novikov V. F., and Mitrofanov D. D. Evaluation of stresses in a presurized vessel by magnetoelastic demagnetization // Diagnostics, Resource and Mechanics of materials and structures. - 2019. - Iss. 6. - P. 6-14. - DOI: 10.17804/24109908.2019.6.006-014. 


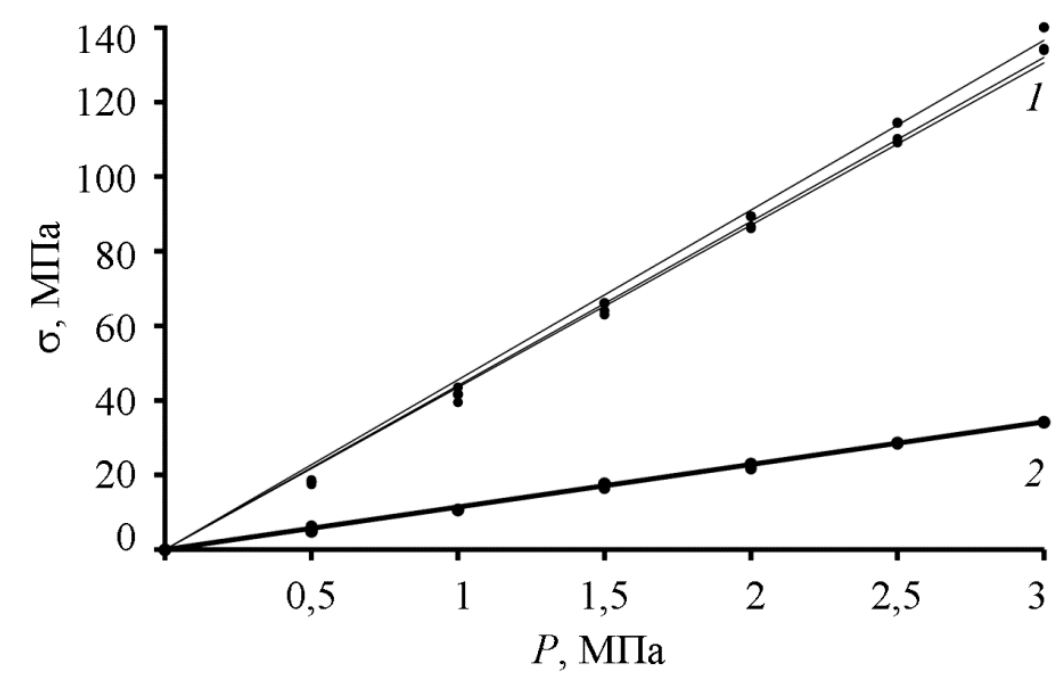

Рис. 1. Зависимость кольцевых $\sigma_{\text {к }}(1)$ и осевых $\sigma_{0}(2)$ механических напряжений в трех точках одной осевой линии стенки газового баллона от внутреннего давления $P$ воды в нем (по результатам тензометрии)

Магнитоупругое размагничивание сложно нагруженной стенки газового баллона исследовалось отдельно при двух способах ее локального намагничивания: вначале вдоль действия кольцевых, затем вдоль осевых деформаций. Для этого использовались два типа перекатываемых $H$ - образных намагничивающих устройств на основе постоянного магнита $\mathrm{SmCo}_{5}$, отличающихся формой полюсных наконечников, позволяющих создавать локально остаточно намагниченное состояние в выделенных областях стенки баллона. Устройство первого типа с полюсными наконечниками в форме диска перемещалось качением по двум кольцевым линиям стенки баллона, расположенным на расстоянии 150 мм друг от друга и намагничивало ее вдоль направления действия осевых напряжений. Для локального намагничивания стенки баллона вдоль действия кольцевых напряжений применялось устройство второго типа, у которого полюсные наконечники были выполнены в форме близкой к усеченному конусу, позволяющей минимизировать воздушный зазор между ними и намагничиваемой цилиндрической поверхностью боковой стенки. Устройство также перемещалось качением вдоль двух параллельных осевых линий стенки, расположенных на расстоянии 180 мм друг от друга. При этом в каждом случае намагничивания формировались по две полосы N-S локальной остаточной намагниченности, ориентированной встречно друг другу. Тем самым исключалось влияние магнитного поля лаборатории и намагниченности конструкции на результаты измерений $[11,15]$. Измерение в максимуме нормальной и тангенциальной составляющих вектора напряженности магнитного поля рассеяния вдоль каждой полосы N-S остаточной намагниченности проводилось феррозондовым магнитометром ИКНМ-2ФП в шести равноудаленных друг от друга точках [11].

\section{3. Результаты и их обсуждение}

Результаты исследования магнитоупругого размагничивания стенки баллона в одной из ее точек, расположенной на пересечении осевой и кольцевой линий показаны на рис. 2.

Как видно наибольшие изменения $\delta H$ напряженности магнитного поля рассеяния локальной остаточной намагниченности сложно нагруженной стенки баллона получены при ее намагничивании вдоль действия кольцевых напряжений (рис. 2 б), создаваемых соответствующей деформацией. Подобные закономерности и отличия размагничивания $\delta H$ были получены для каждой из четырех точек пересечения обеих осевых и кольцевых линий, вдоль которых производилось намагничивание (перемещение намагничивающе- 
го устройства). Обращает на себя внимание, что составляющие напряжений $\sigma_{\kappa}$ и $\delta_{\text {o }}$ отличаются более чем в три раза (рис. 1), тогда как отличия $\delta H$ при соответствующих направлениях намагничивания убывают от четырехкрат до 1,7 при изменении эквивалентных напряжений $\sigma_{э к в}=44 \div 146$ МПа.
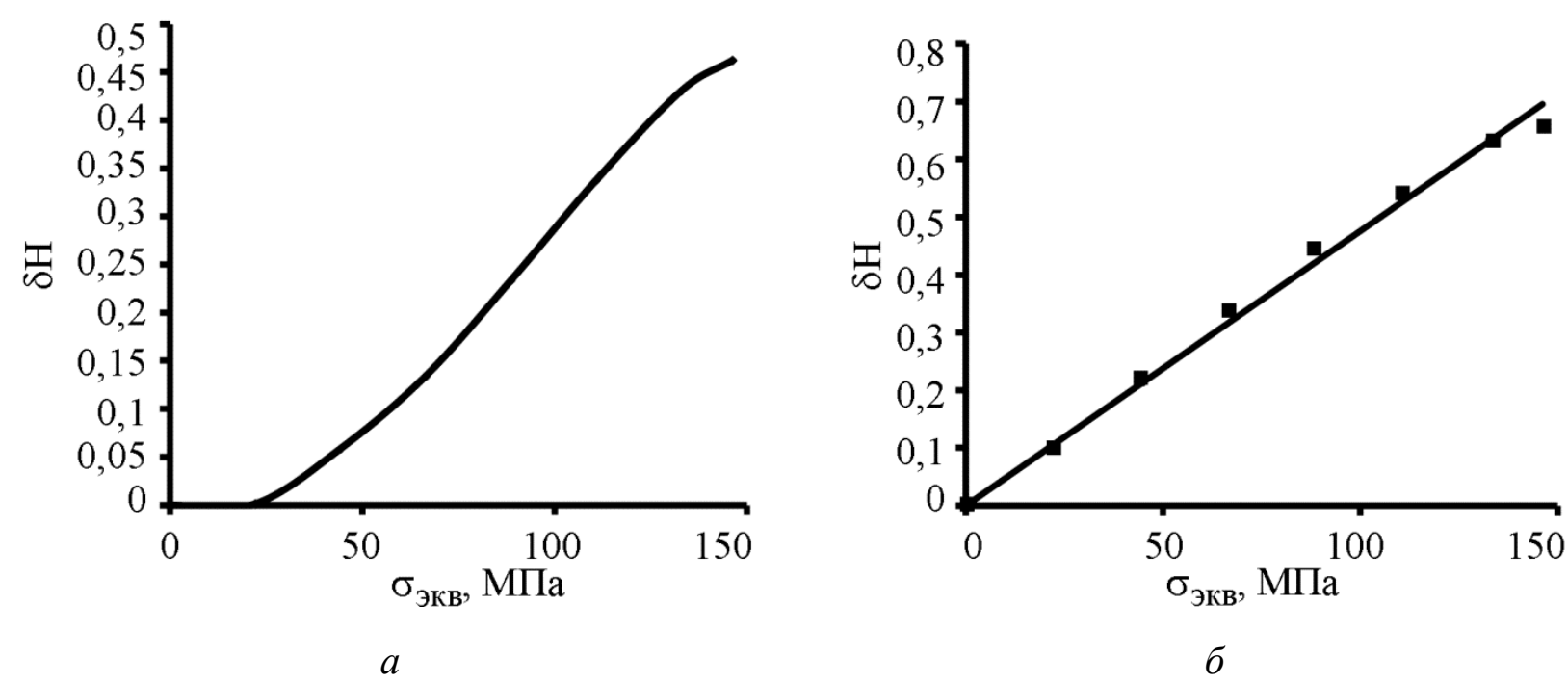

Рис. 2. Зависимость относительного изменения $\delta H$ напряженности магнитного поля рассеяния локальной остаточной намагниченности стенки баллона, намагниченной вдоль $(a)$ и поперек (б) его оси от эквивалентных напряжений $\sigma_{э к в}$, создаваемых действием внутреннего давления

Эффект магнитоупругого размагничивания стали в основном определяется ее гистерезисными свойствами и магнитоупругой энергией. Эффективность перестройки магнитных фаз в результате действия напряжений определяется, как известно, магнитоупругой энергией [18]:

$$
\Delta W_{\mathrm{My}}=-3 / 2 \lambda_{s} \sigma \cos ^{2} \varphi
$$

где $\lambda_{s}-$ константа магнитострикции; $\sigma-$ упругие напряжения; $\varphi-$ средний угол между магнитными моментами доменов и направлением приложения напряжений. Под действием напряжений магнитная система (домены и их границы) перестраивается для достижения минимума ее энергии.

При нагружении внутренним давлением намагниченного вдоль кольца участка стенки баллона напряжение $\sigma_{\text {к }}$ будет стремиться увеличить объем доменов с соосными векторами намагничивания, но так как они уже в основном выстроены магнитным полем вдоль направления напряжения $\sigma_{\mathrm{K}}$ (угол $\varphi$ невелик), то и престройка соосных магнитных фаз и связанное с этим размагничивание за счет этого не будет большим. Вторая компонента сложного напряжения $\sigma_{о}$ будет стремиться переориентировать вектора намагниченности из поперечного в продольное (кольцевое) направление, но так как $\sigma_{\kappa} \gg \sigma_{0}$, то объем магнитных фаз ориентированных поперечно оси не будет большим.

Если вектор остаточной намагниченности стали будет ориентирован вдоль оси цилиндра (в направлении меньшего напряжения $\sigma_{o}$ ), то действие поперечных кольцевых напряжений перестраивая доменную структуру (изменяя ориентацию магнитных моментов в поперечном направлении) вызовет большую перестройку магнитных фаз, по сравнению с осевыми напряжениями, что приведет к увеличению степени магнитоупругого размагничивания и значительному увеличению магнитоупругой чувствительности. В результате кривые 
на рис. 2 а и $2 \sigma$ не смотря на существенную разницу $\sigma_{\mathrm{K}} \gg \sigma_{о}$ сблизятся, а разница в магнитоупругой чувствительности уменьшится.

На рис. 3 а показано распределение эквивалентных напряжений вдоль кольцевой (1) и осевой (2) линий длиной $L=0,5$ м каждая, оцениваемых по величине убыли напряженности магнитного поля рассеяния их остаточной намагниченности в точках с интервалом в 0,1 м (рис. 3 б).

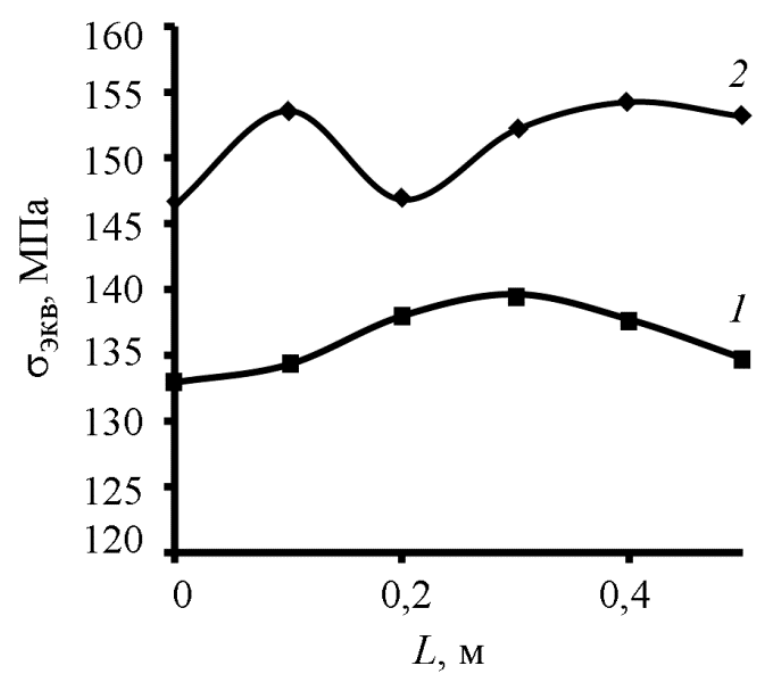

$a$

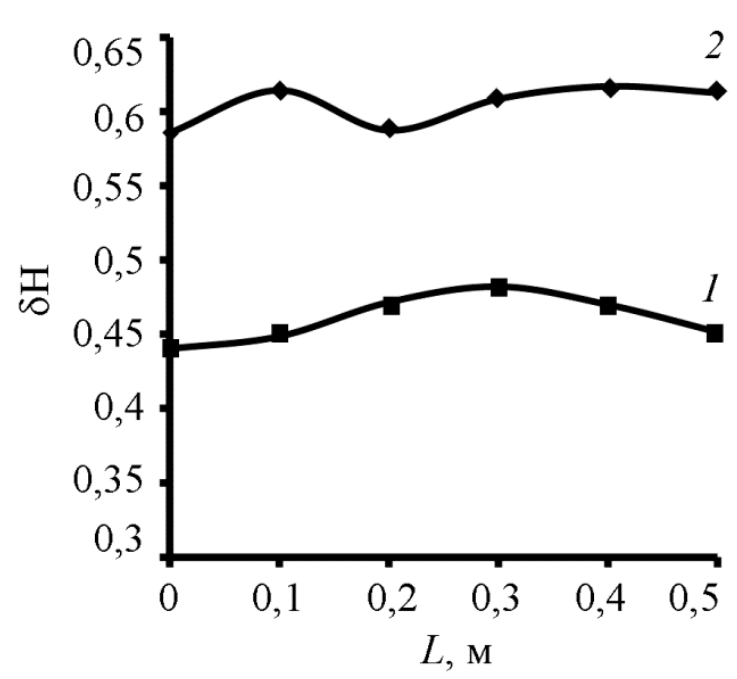

$\sigma$

Рис. 3. Распределение эквивалентных напряжений $(a)$ и относительной убыли напряженности магнитного поля рассеяния остаточной намагниченности (б) вдоль кольцевой (1) и осевой (2) линий боковой стенки баллона при максимальном внутреннем давлении воды $P=3,3$ МПа

Как видно из рис. 3 а уровень эквивалентных напряжений вдоль кольцевой линии стенки баллона изменяется от 133 до 139 МПа, а вдоль осевой линии от 147 до 153 МПа. Разный уровень магнитоупругого размагничивания (рис. 3 б) в шести точках как осевой, так и кольцевой линий при условии однородности напряженного состояния баллона объясняется отличием магнитоупругой чувствительности стали в них. Учет величины магнитоупругой чувствительности стали в каждой контролируемой точке конструкции позволит повысить точность оценки уровня механических напряжений магнитоупругим методом.

В каждой из шести точек, выбранных для измерения поля остаточной намагниченности осевых и кольцевых N-S полос стенки баллона производился также контроль коэрцитивной силы $H_{\mathrm{c}}$ при увеличении внутреннего давления $P$ воды в нем. При этом для исключения влияния внутреннего магнитного поля стальной стенки на результаты этих измерений полюсные наконечники блока коэрцитиметра КРМ - Ц - К2М располагались вдоль кольцевой линии, производилось измерение $H_{\mathrm{c}}$, блок разворачивался на $180^{\circ}$ и измерение повторялось, результат которого затем усреднялся с полученным до этого. Аналогичным

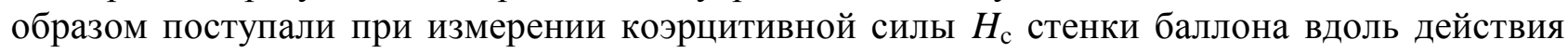
осевых напряжений.

Результаты измерения коэрцитивной силы стенки газового баллона в той же точке, что и рис. 2. показан на рис. 4. Видно, что анизотропия коэрцитивной силы растет вследствие неодинаковости анизотропии составляющих напряжений (рис. 5). Убыль коэрцитивной силы, измеренной вдоль действия наращиваемых кольцевых напряжений превышает ее прирост, вдоль направления действия увеличиваемых осевых напряжений. При этом результат измерения $H_{\mathrm{c}}$ баллона в исходном (не нагруженном состоянии) заметно отличается от значений, полученных в работах $[10,13]$ на стали Ст3, из которой согласно [16] должны изготавливаться такие конструкции. 


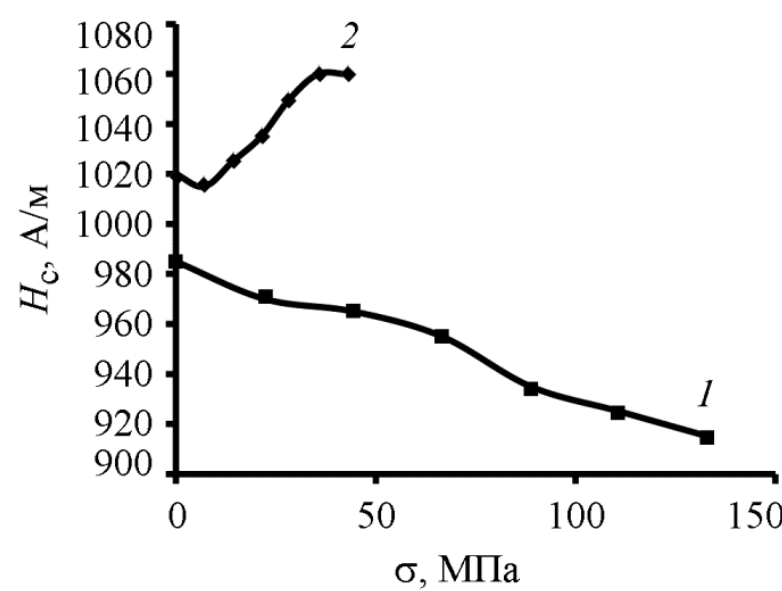

Рис. 4. Зависимость $H_{\mathrm{c}}$ измеренной вдоль кольца от кольцевых $\sigma_{\kappa}$ напряжений (1) и $H_{\mathrm{c}}$ измеренной вдоль оси от осевых $\sigma_{\mathrm{o}}$ напряжений (2)

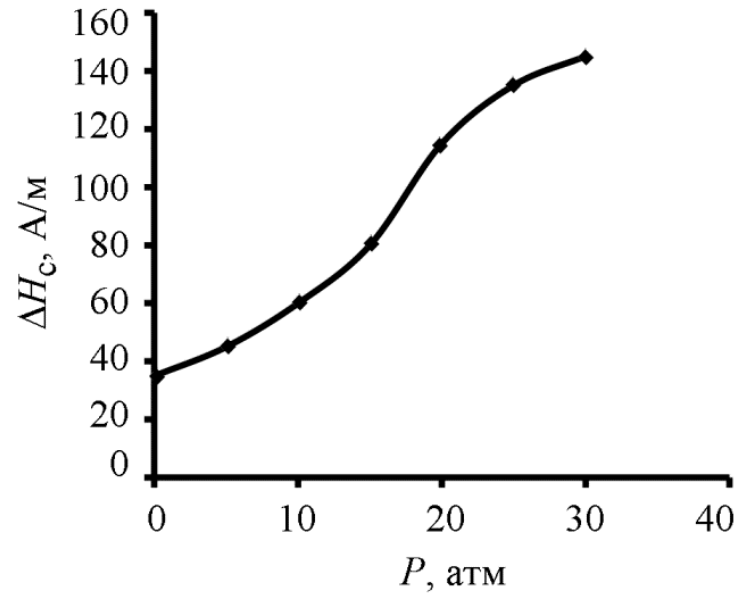

Рис. 5. Зависимость разности (анизотропии) коэрцитивной силы $H_{\mathrm{c}}$ измеренной вдоль оси и кольца баллона от внутреннего давления в нем

Видно, что коэрцитивная сила $H_{\mathrm{c}}$, измеренная вдоль оси баллона возрастает на $4 \%$

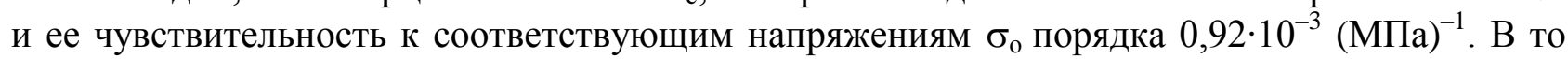
время как изменение $H_{\text {c }}$ стальной стенки баллона измеренной вдоль действия кольцевых

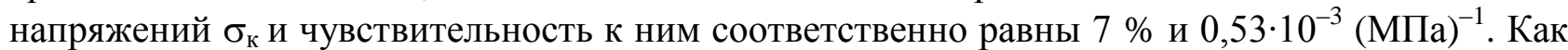
известно [7] в поперечном направлении при одноосной деформации стали $H_{\mathrm{c}}$ только возрастает, а в продольном вначале уменьшается, а затем возрастает. При двухосном ортогональном растяжении стенки газового баллона создается анизотропия механических напряжений, формирующих анизотропию магнитной текстуры ферромагнетика (стали) с преобладанием в ней магнитных моментов ориентированных вдоль кольцевого направления, поскольку $\sigma_{\mathrm{K}}>\sigma_{0}$, а, следовательно, и уменьшению напряженности внешнего магнитного поля (т. е. $H_{\mathrm{c}}$ ), необходимого для перемагничивания стенки в этом направлении. Изменение разности значений коэрцитивной силы $\Delta H_{\mathrm{c}}$, измеряемой соосно главным действующим в стенке баллона напряжениям $\sigma_{\mathrm{K}}$ и $\sigma_{\mathrm{o}}$ с ростом величины внутреннего давления $P$ показано на рис. 5 .

\section{4. Заключение}

Установлено, что анизотропная локальная намагниченность стенки баллона, ориентированная вдоль действия осевых напряжений, создаваемых внутренним давлением убывает на $30 \%$ медленнее по сравнению с ортогональной к ней локальной намагниченностью ориентированной вдоль кольцевых напряжений.

Магнитоупругая чувствительность остаточной намагниченности сложно нагруженной в упругой области стали заметно выше чувствительности ее коэрцитивной силы, что позволяет с большей разрешающей способностью контролировать распределение напряжений в сосудах с давлением.

\section{Литература}

1. ГОСТ Р 56542-2015. Национальный стандарт Российской Федерации. Контроль неразрушающий. Классификация видов и методов / введ. 2016-06-01. - М. : Стандартинформ, 2019.

2. The effect of elastic de-formations on the magnetic properties of Chromium-Nickel steels / E. S. Gorkunov, E. I. Yakushenko, S. M. Zadvorkin, A. N. Mushnikov // Physics of Metals and Metallography. - 2015. - Vol. 116, no. 2. - P. 156-164. - DOI 10.7868/S0015323015020072. 
3. Неразрушающий контроль : справочник : в 7 т. / под общ. ред. В. В. Клюева. Т. 6 : в 3 кн. Кн. 1 : Магнитные методы контроля / В. В. Клюев, В. Ф. Мужицкий, Э. С. Горкунов, В. Е. Щербинин. - М. : Машиностроение, 2004. - 832 с.

4. Неразрушающий контроль. Магнитные методы контроля. Т. 6, кн. 1 / В. В. Клюев, В. Ф. Мужицкий, Э. С. Горкунов, В. Е. Щербинин. - Москва : Машиностроение, 2006. - 375 с.

5. Birss R. B., Fauncer C. A., Isaak E. D. Magneto-mechanical effects in iron and iron-carbon alloys // J. Appl. Phys. - 1971. - Vol. 4. - P. 1040-1048. - DOI: 10.1088/0022-3727/4/7/322.

6. Atherton D. L., Jiles D. C. Effects of stress on the magnetizatio $n$ of steel // IEEE Trans. Magn. - 1983. - Vol. 19, iss. 5. - P. 2021-2023. - DOI: 10.1109/TMAG.1983.1062784.

7. Novikov V. F., Yatsenko T. A., Bakharev M. S. // Rus. J. Nondestr. Test. - 2001. - Vol. 37, no. 11. - P. 799-804 // Rus. J. Nondestr. Test. - 2002. - Vol. 38, no. 4. - P. 231-237.

8. Irreversible Changes in the Magnetization as Indicators of Stress-strain State of Ferromagnetic Objects / V. N. Kostin, T. P. Tsarkova, A. P. Nichipuruk, V. E. Loskutov, V. V. Lopatin, K. V. Kostin // Rus. J. Nondestr. Test. - 2009. - Vol. 45, no. 11. - P. 786-798. DOI: $10.1134 / \mathrm{S} 1061830909110059$.

9. Zakharov V. A., Ul'yanov A. I., Gorkunov E. S. Regularities of the change in the coercive force under biaxial asymmetric deformation of steel 3 // Russian Journal of Nondestructive Testing. 2010. - Vol. 46, no. 3. - P. 194-205. - DOI: 10.1134/S1061830910030071.

10. The influence of biaxial elastic deformation on the coercive force and local remanent magnetization of construction steels / V. F. Novikov, S. V. Sorokina, M. E. Kudryashov, V. A. Zakharov, and A. I. Ul'yanov // Russ. J. Nondestr. Test. - 2010. - Vol. 46, no. 7. - P. 520-526. DOI: $10.1134 / \mathrm{S} 1061830910070065$.

11. Magnetic Testing of Stressed State of Hydrotested Gas-Separator Wall / S. M. Kulak, V. F. Novikov, V. V. Probotyuk, S. M. Vatsenkov, E. S. Fursov // Russian Journal of Nondestructive Testing. - 2019. - Vol. 55, no. 3. - P. 225-232. - DOI: 10.1134/S1061830919030070.

12. Muzhitskii V. F., Popov B. E., and Bezlyud'ko G. Ya. Magnetic Measurements of StressedStrained States and Remaining Service Lives of Steel Structures in Hoisting Machines and Pressurized Vessels // Russ. J. Nondestr. Test. - 2001. - Vol. 37, no. 1. - P. 29-36.

13. Zakharov V. A., Ul'yanov A. I., and Gorkunov E. S. Coercive Force of Ferromagnetic Steels under Biaxial Symmetric Tension of Materials // Rus. J. Nondestr. Test. - 2011. - Vol. 47, no. 6. - P. 359-368. - DOI: 10.1134/S1061830911060106.

14. On controlling stresses in a complexly loaded steel construction by magnetoelastic demagnetization / V. F. Novikov, V. P. Ustinov, A. V. Radchenko, K. R. Muratov, S. M. Kulak, S. V. Sorokina // Russian Journal of Nondestructive Testing. - 2016. - Vol. 52, no. 6. - P. 357-361. DOI: $10.1134 / \mathrm{S} 1061830916060073$.

15. Novikov V. F., Prilutskii V. V. The properties of stripe-shaped residual magnetization and the possibilities for its application for nondestructive testing // Russian Journal of Nondestructive Testing. - 2014. - Vol. 50. - P. 396-401. - DOI: 10.1134/S1061830914070080.

16. ГОСТ 15860-84. Баллоны стальные сварные для сжиженных углеводородных газов на давление до 1,6Мпа / введ. 1985-07-01. - Москва : ИПК Издательство стандартов, 1984.

17. Бородавкин П. П., Синюков А. М. Прочность магистральных трубопроводов. - М. : Недра, 1984. - 248 с.

18. Вонсовский С. В., Шур Я. С. Ферромагнетизм. - М.-Л. : ГИТТЛ, 1948. - 816 с. 